\title{
Extensive intrathecal T cell renewal following hematopoietic transplantation for multiple sclerosis
}

Kristina M. Harris, ${ }^{1}$ Noha Lim, ${ }^{1}$ Paul Lindau, ${ }^{2}$ Harlan Robins, ${ }^{2,3}$ Linda M. Griffith, ${ }^{4}$ Richard A. Nash, ${ }^{5}$ Laurence A. Turka, ${ }^{1,6}$ and Paolo A. Muraro ${ }^{7}$

IImmune Tolerance Network, Bethesda, Maryland, USA. 'Fred Hutchinson Cancer Research Center, Seattle, Washington, USA. ${ }^{3}$ Adaptive Biotechnologies, Seattle, Washington, USA. ${ }^{4}$ Division of Allergy, Immunology and Transplantation, National Institute of Allergy and Infectious Diseases, NIH, Bethesda, Maryland, USA. ${ }^{5}$ Colorado Blood Cancer Institute, Presbyterian/St. Luke's, Denver, Colorado, USA. 'Department of Surgery, Massachusetts General Hospital, Boston, Massachusetts, USA. `Department of Brain Sciences, Imperial College London, London, United Kingdom.

A recent study of autologous hematopoietic stem cell transplantation (AHSCT) for active relapsing-remitting multiple sclerosis (RRMS) showed efficacy in preventing disease worsening. However, the immunologic basis for efficacy remains poorly defined. Multiple sclerosis pathology is known to be driven by inflammatory T cells that infiltrate the CNS. Therefore, we hypothesized that the preexisting $\mathrm{T}$ cell repertoire in the intrathecal compartment of active RRMS participants was ablated and replaced with new clones following AHSCT. T cell repertoires were assessed using high-throughput TCR $\beta$ chain sequencing in paired cerebrospinal fluid (CSF) and peripheral blood $\mathrm{CD4}^{+}$and $\mathrm{CD}^{+} \mathrm{T}$ cells from participants that underwent AHSCT, before and up to 4 years following transplantation. More than $90 \%$ of the preexisting CSF repertoire in participants with active RRMS was removed following AHSCT and replaced with clonotypes predominantly generated from engrafted autologous stem cells. Of the preexisting clones in CSF, approximately $60 \%$ were also detected in blood before therapy, and concordant treatment effects were observed for clonotypes in both compartments following AHSCT. These results indicate that replacement of the preexisting TCR repertoire in active RRMS is a mechanism for AHSCT efficacy and suggest that peripheral blood could serve as a surrogate for CSF to define mechanisms associated with efficacy in future studies of AHSCT.

Conflict of interest: LAT is employed by, and holds equity grants in, Rheos Medicines. HR is employed by and holds equity in Adaptive Biotechnologies.

Copyright: ( 2020 , American Society for Clinical Investigation.

Submitted: February 2, 2019 Accepted: December 18, 2019 Published: January 30, 2020.

Reference information: /CI Insight. 2020;5(2):e127655.

https://doi.org/10.1172/jci. insight.127655.

\section{Introduction}

Autologous hematopoietic stem cell transplantation (AHSCT) has been investigated in clinical trials for aggressive autoimmunity as a therapeutic strategy for replacing the autoreactive immune system with a new, potentially self-tolerant immune repertoire (1). High-Dose Immunosuppression and Autologous Transplantation for Multiple Sclerosis (HALT-MS) was a phase II study of AHSCT for patients with treatment-resistant relapsing-remitting multiple sclerosis (RRMS), which showed that AHSCT without maintenance disease-modifying therapy was effective for inducing durable remissions of active RRMS for at least 5 years (2). In the cohort of 24 participants that underwent AHSCT, 17 achieved durable complete remission from active disease, 3 experienced clinical relapse, 2 showed disease progression by increased Expanded Disability Status Scale, and 2 had recurrence of CNS inflammation detected by MRI through the 60 months follow-up (2). Notably, all study participants showed an attenuation of disease activity after AHSCT compared with before therapy.

In 2014, we used high-throughput T cell receptor (TCR) sequencing to analyze how AHSCT modified the peripheral blood repertoire in patients enrolled in the HALT-MS trial. This study showed that, while the CD4 repertoire was largely replaced as a result of the procedure, the CD8 repertoire was less profoundly affected, as many preexisting CD8 T cell clones "survived" the procedure and expanded at month 12 after transplant (3). While providing novel information about the effects of AHSCT on the immune repertoire, this interim analysis was limited to what was seen in peripheral blood at 12 months after transplant and provided no insight into what was happening in the target organ of the disease, the CNS. 
Multiple sclerosis (MS) pathology is known to be driven by inflammatory T cells that enter and infiltrate the CNS (4). T cells that access the CNS recirculate in the cerebrospinal fluid (CSF) (5), thus presenting a more direct point of access to the site of disease than peripheral blood (6). However, the sampling limitations and the low number and instability of $\mathrm{T}$ cells in CSF have hampered the ex vivo characterization of these cells (7). To investigate one mechanism potentially explaining the strong mitigating effect on CNS inflammation seen in HALT-MS, we hypothesized that the T cell repertoire in the intrathecal compartment before therapy, likely including autoreactive $\mathrm{T}$ cells, was ablated and replaced with new clones after AHSCT therapy. To test this, we have compared the reconstitution of T cell composition in peripheral blood to paired reconstitution in the target disease organ using CSF from HALT-MS participants analyzed by high-throughput deep sequencing of TCR $\beta$ complementary determining region 3 (CDR3) chains $(7,8)$.

The data reported herein support the hypothesis that AHSCT removes the majority of $\mathrm{T}$ cell clones present in the intrathecal compartment of patients with active RRMS and enables the generation of a new immune repertoire in both intrathecal and peripheral blood compartments. In addition, our findings suggest that peripheral blood could serve as a surrogate for CSF to define mechanisms associated with efficacy of AHSCT in active RRMS. These potentially novel and important insights identify avenues of investigation for future studies of AHSCT and other immunomodulatory intervention strategies for MS.

\section{Results}

Persistent ablation of preexisting the T cell repertoire in peripheral blood following AHSCT therapy. We previously reported that AHSCT had distinctive effects on $\mathrm{CD}^{+}$and $\mathrm{CD} 8^{+} \mathrm{T}$ cell repertoires in peripheral blood of HALT-MS participants (3). In circulating $\mathrm{CD}^{+} \mathrm{T}$ cells, participants largely developed a new repertoire, whereas the reconstituted $\mathrm{CD} 8^{+} \mathrm{T}$ cell repertoire was created by clonal expansion of preexisting cells that were not effectively removed by AHSCT therapy (3). We have extended this analysis to evaluate the longer-term reconstitution of TCR repertoires in blood $\mathrm{CD}^{+}$and $\mathrm{CD}^{+} \mathrm{T}$ cell compartments using an approach that could be applied to the corresponding CSF samples. The percentage of TCR overlap was used to determine what $\mathrm{T}$ cell repertoires were identical in identical tissues (blood or CSF) at different time points. This metric is preferred because it ensures a method robust to differences in sampling depth by weighting the relative frequencies of shared clones between time points (8).

Consistent with our previous report (3), most of the preexisting $\mathrm{CD}^{+} \mathrm{T}$ cell repertoire in blood before therapy was undetectable by ultra-deep sequencing in blood following transplantation at months 12,24 and 48 (mean $81.4 \% \pm 7.4 \%$ SD at month 12 ; mean $83.8 \% \pm 5.5 \%$ SD at month 24 ; mean $88.7 \% \pm 4.7 \%$ SD at month 48) (Figure 1). In blood, ablation of the preexisting $\mathrm{CD} 8^{+} \mathrm{T}$ cell repertoire was significantly less extensive compared with the $\mathrm{CD} 4{ }^{+} \mathrm{T}$ cell compartment $(P<0.001, P<0.001$, and $P<0.01$ at months 12,24 , and 48 , respectively) and was highly variable among participants (mean $68.2 \% \pm 12.8 \%$ SD at month 12 ; mean $70.9 \% \pm 11.9 \%$ SD at month 24 ; mean $71.2 \% \pm 15.2 \%$ SD at 48 ) (Figure 1). The striking changes we observed in the TCR repertoire of transplanted patients were almost certainly due to AHSCT therapy itself and not the interval between sample time points, given the repertoire stability we previously reported for paired samples of T cells collected from healthy individuals 12 months apart (3). These data show that the effects of AHSCT on the elimination of preexisting $\mathrm{T}$ cell repertoire in peripheral blood are durable through 48 months follow-up.

AHSCT therapy removes most of the preexisting $T$ cell repertoire in CSF. T cells recirculating in the intrathecal compartment provide a more direct point of access to the CNS than peripheral blood $(5,6)$ and may therefore contain a higher frequency of disease-causing clonotypes than peripheral blood. Thus, we asked whether elimination of T cell clones from the CSF of MS patients could be one of the mechanisms by which AHSCT effectively treated RRMS in HALT-MS (2). To determine the sharing between T cell repertoires in CSF before therapy versus time points after transplant, the percentage of TCR overlap was used because it ensures a method robust to differences in sampling depths between time points (Supplemental Tables 1 and 3; supplemental material available online with this article; https://doi.org/10.1172/ jci.insight.127655DS1) by weighting the relative frequencies of shared clones between time points (8). The majority of $\mathrm{T}$ cells detected in CSF before therapy were undetectable in CSF by deep sequencing at months 24 and 48 after transplant (Figure 2A). On average, $91.8 \%( \pm 7.2 \% \mathrm{SD})$ and $93.5 \%( \pm 6.9 \% \mathrm{SD})$ of the preexisting CSF repertoire was undetectable in CSF at 24 and 48 months, respectively. Abundance of preexisting clones in CSF before therapy was not associated with whether or not clonotypes were detected or undetectable in CSF at month 24 after transplant $(P=0.4)$ (Figure 2B), but indeed some clones were highly persistent following AHSCT. These data indicate that most of the preexisting TCR repertoire in 


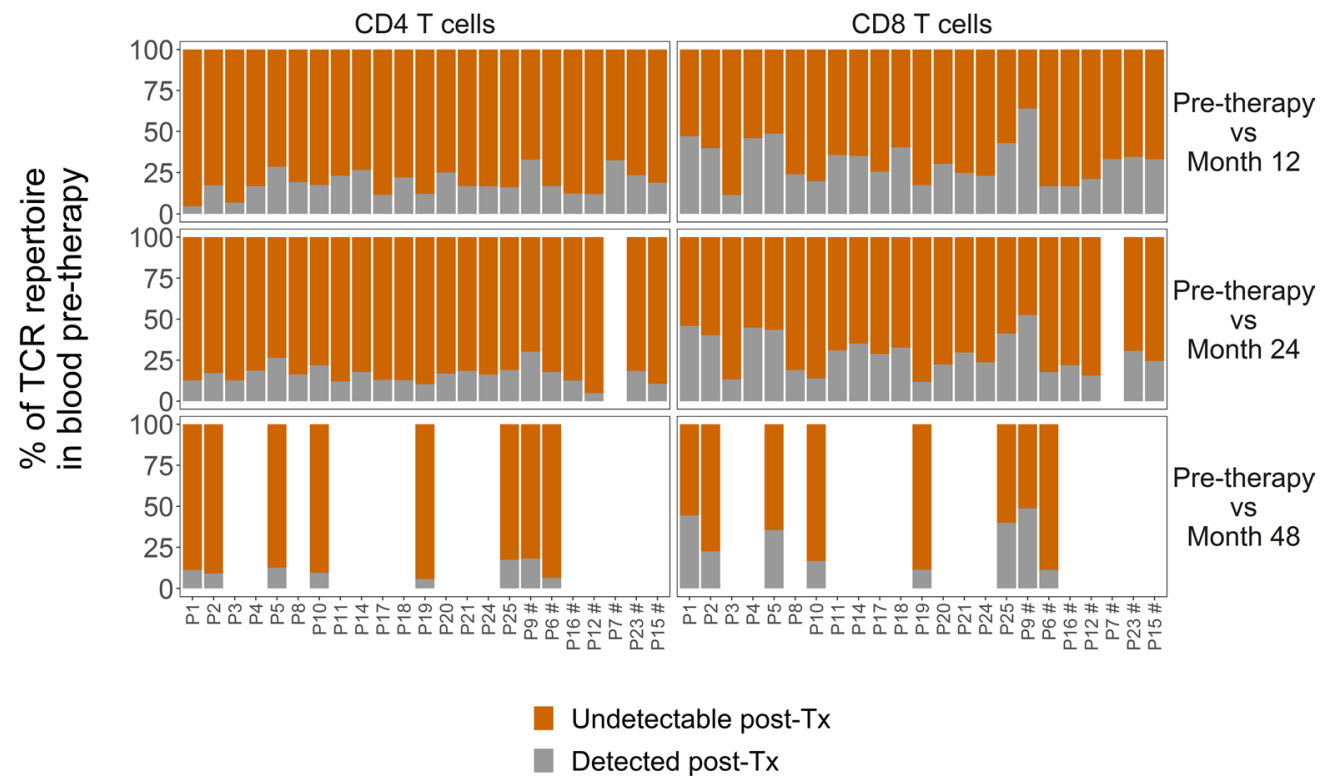

Figure 1. Effects of AHSCT therapy on ablation of preexisting T cell repertoires in peripheral blood are durable through 48 months. TCR overlap percentage was used to determine the percentage of clones that were undetectable (orange) versus detected (gray) by ultra-deep sequencing of TCR repertoires in CD4+ $\mathrm{T}$ cells and CD8+ $\mathrm{T}$ cells isolated from peripheral blood, comparing before therapy to months 12, 24, or 48 for each participant. \#Participants that met the primary endpoint for the HALT-MS study. All other participants remained in remission from active MS until the last follow-up.

CSF was removed by AHSCT irrespective of the clones' individual frequency and show that the effects are durable through 48 months follow-up.

We next examined the relationship between clones shared between the CSF and peripheral blood compartments in active RRMS before therapy and at month 24 after AHSCT therapy. On average, $62.3 \%$ $( \pm 6.1 \% \mathrm{SD})$ of clones in CSF before therapy were also detected in blood before therapy (Figure $2 \mathrm{C}$ ). The degree of clonal sharing between the CSF and peripheral blood compartments was significantly reduced following AHSCT at month 24 after transplant to $40.6 \%$ ( $\pm 7 \%$ SD) $(P<0.0001$, Figure $2 \mathrm{C})$. Given the efficacy of AHSCT in this study cohort, we asked whether AHSCT removed clones exclusively detected in CSF before therapy. Our rationale was that clonotypes enriched or exclusively detected in CSF of patients with active RRMS (as per trial inclusion criteria) may comprise a higher frequency of $\mathrm{T}$ cells with specificity to CNS antigens, likely including disease-causing $\mathrm{T}$ cells, than would be detected in peripheral blood. We found that $40.5 \%( \pm 11.8 \% \mathrm{SD})$ of preexisting clones that were removed from the CSF following AHSCT were exclusively detected in CSF (Figure 2D, gold vs. red bars), whereas clones that persisted in CSF were predominantly shared between compartments before therapy (Figure 2D, gray vs. blue bars). Notably, very few clones that were detected in CSF and not in blood before therapy persisted at 24 months, which indicates that $\mathrm{T}$ cell clones detected in CSF after transplant originate from precursors in peripheral blood.

$T$ cell renewal in CSF following AHSCT therapy. Having determined that $>90 \%$ of the $\mathrm{T}$ cell repertoire in CSF before therapy was undetectable at months 24 or 48 after AHSCT therapy (Figure 2A), we wanted to determine the proportion of new $\mathrm{T}$ cell clones arising in the reconstituted CSF repertoire following AHSCT therapy. The percentage of TCR overlap analysis showed that the majority $(80.3 \% \pm 14.2 \% \mathrm{SD})$ of T cells detected in CSF at month 24 were undetectable by deep sequencing in the CSF before therapy (Figure 3A), indicating that most of the reconstituted repertoire in the intrathecal compartment comprised new species of $\mathrm{T}$ cell clones. To ensure the robust changes observed were not due to differences in sampling depths between time points (Supplemental Tables 1 and 3), randomized resampling was performed 1000 times on pretherapy CSF TCR $\beta$ sequences to match with the corresponding sampling of CSF TCR $\beta$ sequences at month 24 after transplant. The mean percentage of clones that overlapped in smaller posttransplant sampling from the larger pretherapy samples was determined to be $92.9 \%$ ( $\pm 6.5 \% \mathrm{SD}$ ) (Supplemental Figure 1). These results rule out sampling bias and strengthen the claim that AHSCT replaced $>90 \%$ of the preexisting $\mathrm{T}$ cell repertoire in CSF with new clones at month 24 after transplant (Figure 2A). 
A

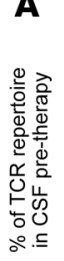

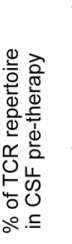

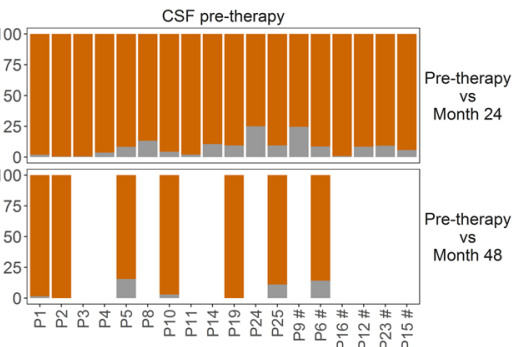

Undetectable post-Tx

Detected post-TX

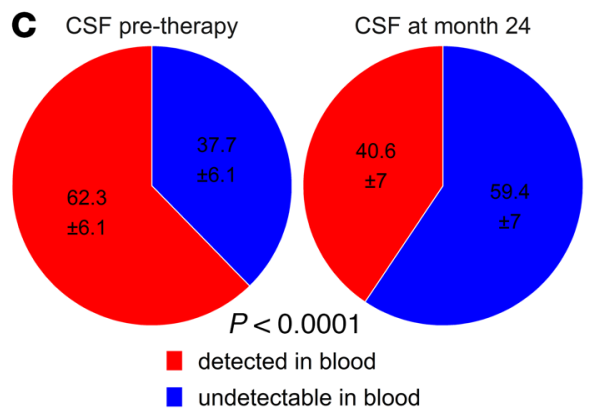

B

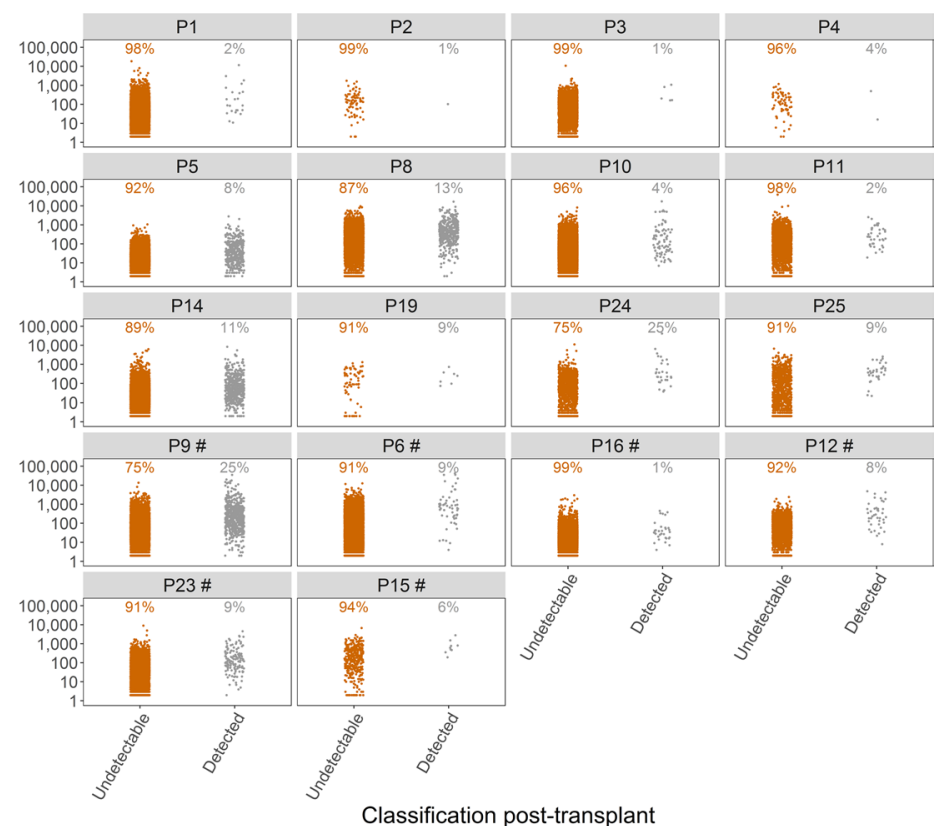

D CSF pre-therapy

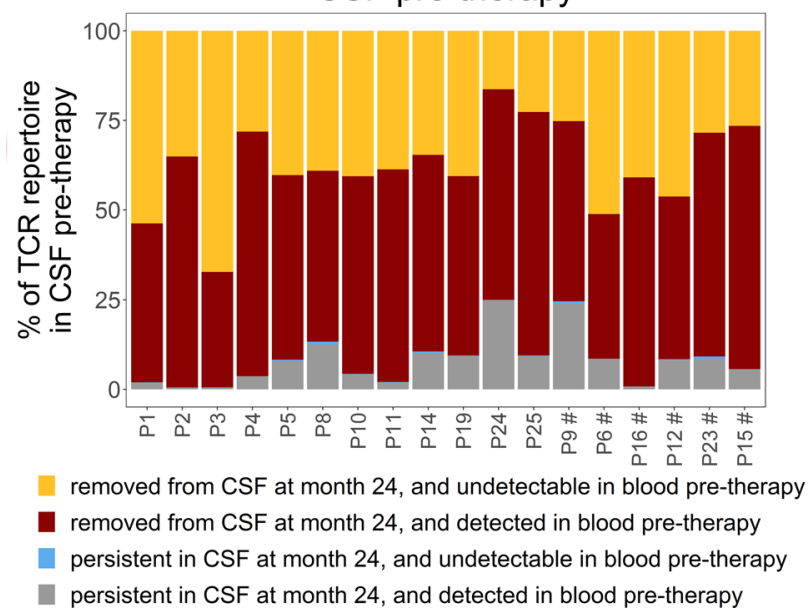

Figure 2. The preexisting CSF T cell repertoire was substantially removed following AHSCT therapy. (A) TCR overlap percentage was used to determine the percentage of clones that were undetectable (orange) versus detected (gray) by deep sequencing of TCR repertoires in CSF, comparing before therapy to months 24 or 48 for each participant. (B) Number of TCR reads for each clone in CSF before therapy that was undetectable (orange) or detected (gray) in CSF at month 24 for each participant. Percentages correspond to the relative proportion of the TCR repertoire in CSF before therapy in A. (C) Percentage of clones in CSF that were either detected (red) or undetectable (blue) in peripheral blood CD4+ or CD8 ${ }^{+}$T cells before therapy versus at month 24 after transplant. $P<0.0001$, percentage of CSF clones detected in blood at month 0 before therapy versus percentage of CSF clones detected in blood at month 24 after transplant using paired $t$ test. (D) TCR overlap analysis was used to determine the percentage of clones that were undetectable versus detected in the TCR repertoire in CSF before therapy compared with month 24 after transplant, as in $\mathbf{A}$. The percentage of these clones that were detected versus undetectable in pretherapy peripheral blood CD4 $4^{+}$or $C D 8^{+} T$ cells was determined by ultra-deep sequencing, and clones in CSF before therapy were classified into 1 of 4 categories: removed from CSF at month 24 and either undetectable in blood before therapy (gold) or detected in blood before therapy (red); persistent in CSF at month 24 and either undetectable in blood (blue) or detected in blood before therapy (gray). "Participants that met the primary endpoint for the HALT-MS study. All other participants stayed in remission from active MS until the last follow-up.

Clonotypes identified as new in the reconstituted CSF could arise from mature $\mathrm{T}$ cells that survived the immunoablative procedure and were preexisting in blood but not in CSF or from differentiation of engrafted autologous hematopoietic stem cells (aHSCs). To address this, the percentage of new T cell clones in CSF at month 24 after transplant that were detected versus undetectable by ultra-deep sequencing in pretherapy blood samples was determined for individual participants. The rationale was that new clones in reconstituted CSF that were detected in blood before therapy were generated from preexisting $\mathrm{T}$ cells that survived immunoablation, whereas new clones arising in CSF that were undetectable in blood 
A

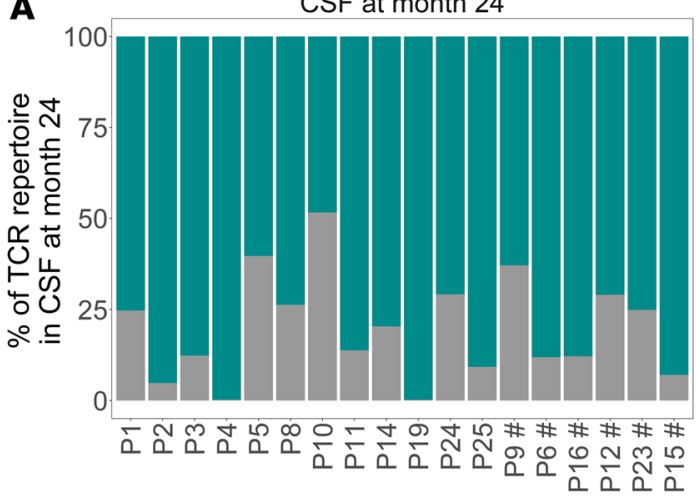

New post-Tx | Persistent post-Tx

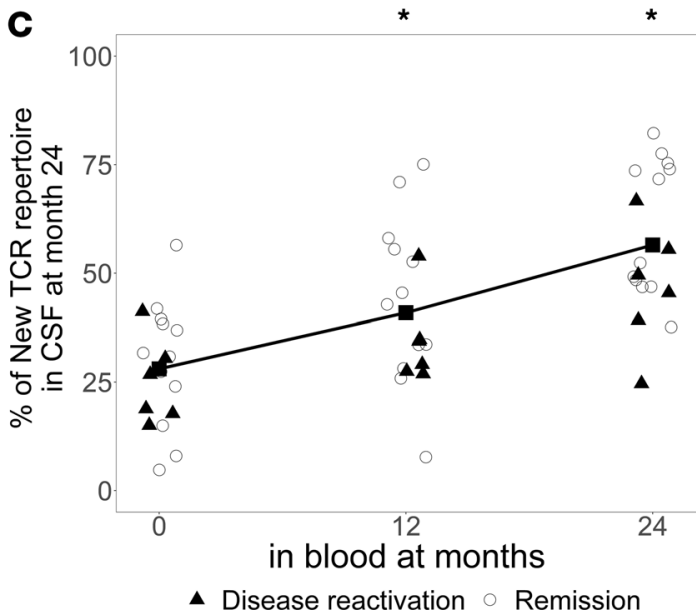

B

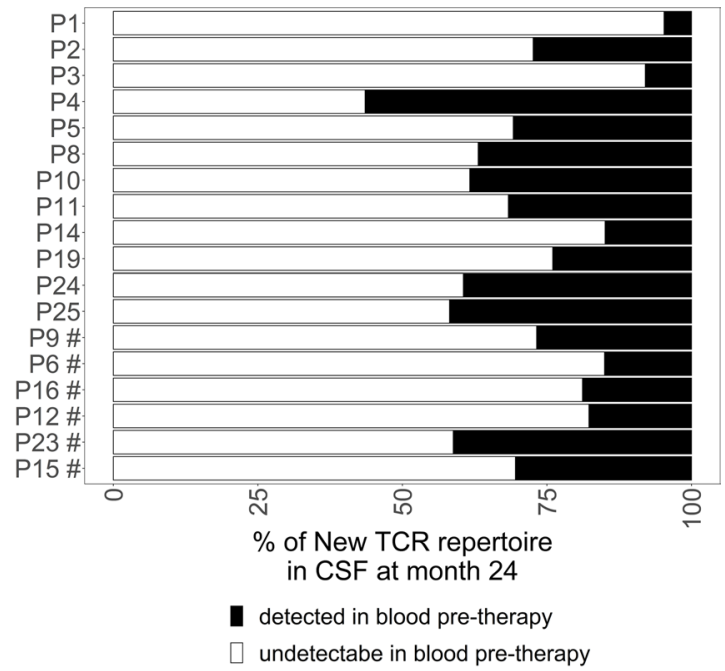

Figure 3. The CSF T cell repertoire was substantially replaced following AHSCT therapy with clonotypes originating predominantly from the graft. (A) TCR overlap percentage was used to determine the percentage of clones that were undetectable, termed new (teal), versus detected, termed persistent (gray), in the CSF repertoire at month 24 compared with before therapy for each participant. (B) The percentage of new clones in CSF at month 24 that were detected (black) versus undetectable (white) by ultra-deep sequencing in pretherapy peripheral blood $\mathrm{CD} 4^{+}$or $\mathrm{CD} 8^{+} \mathrm{T}$ cells was determined. \#Participants that met the primary endpoint for the HALT-MS study. All other participants stayed in remission from active MS until the last follow-up. (C) Longitudinal evaluation of new clones in CSF at month 24 as the percentage detected by ultra-deep sequencing in peripheral blood CD4 ${ }^{+}$or CD8 ${ }^{+} \mathrm{T}$ cells at the indicated time points. Black triangles represent participants that met the primary endpoint for the HALT-MS study before month 60 after transplant, and white circles represent participants that stayed in remission from active MS until the last follow-up. The line represents the mean of participants evaluated. ${ }^{*} P<0.05$, between month 0 before therapy versus months 12 and 24 after transplant using mixed model for repeated measures.

before therapy likely originated from new differentiation. Most (mean $72 \% \pm 13.3 \% \mathrm{SD}$ ) of the new $\mathrm{T}$ cell repertoire in CSF at month 24 after transplant comprised clonal species that were undetectable in blood before therapy for all participants evaluated with the exception of P4 and, thus, likely generated from $\mathrm{T}$ cell differentiation of engrafted aHSCs (Figure 3B). As new $\mathrm{T}$ cell clones differentiate from engrafted aHSCs and repopulate the peripheral blood repertoire, and the CSF is populated by continuous trafficking of blood-circulating cells, one might expect that the proportion of new clones arising in CSF at month 24 after transplant that are also detected in blood should increase over time. Consistent with this notion, the percentage of $\mathrm{T}$ cell clones identified as new in CSF at month 24 after transplant that were also detected in blood was significantly higher at months 12 (mean 40.9\% $\pm 17.3 \mathrm{SD}$ ) and 24 (mean $56.5 \% \pm 16.4 \mathrm{SD}$ ) after transplant as compared with month 0 before therapy (mean $28 \% \pm 13.3$ SD) (Figure 3C and Supplemental Figure 2).

Longitudinal evaluation in blood of clones detected in both CSF and peripheral blood T cell compartments. Having observed extensive removal of preexisting clonotypes in both CSF (Figure 2A) and CD4 T cells in blood (Figure 1) following hematopoietic transplantation, together with the notable degree of clonal sharing between CSF and blood compartments before therapy (Figure 2C), we wanted to determine if the treatment effects observed in CSF were reflected in T cells in blood. To assess this, clones that were classified as removed, new, or persistent in CSF at month 24 after transplant, which were also detected in blood before 

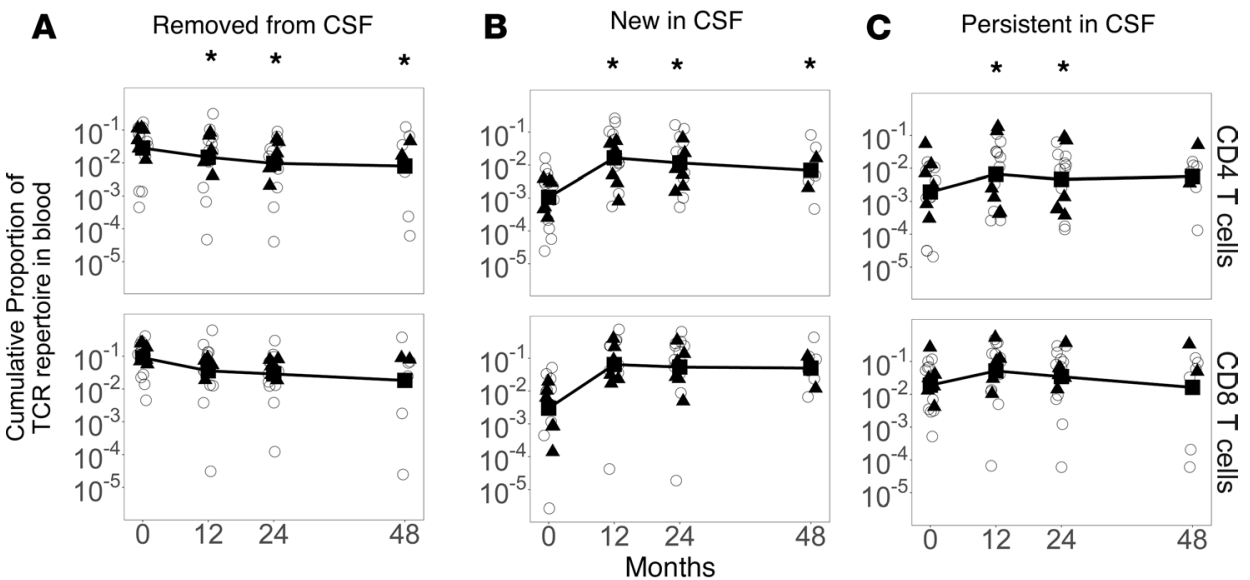

$\Delta$ Disease reactivation - Remission

Figure 4. Longitudinal evaluation in blood of clonotypes detected in both CSF and CD4+ ${ }^{+}$or CD8 ${ }^{+} \mathrm{T}$ cells in blood. Clonotypes classified as (A) preexisting in CSF before therapy and undetectable in CSF at month 24, referred to as removed from CSF, (B) new in CSF at month 24 after transplant, and (C) persisting in CSF at month 24 after transplant. Proportions were aggregated per subject within circulating $C D 4^{+}$or $C D 8^{+} T$ cell repertories before-to-after transplant and then log transformed. Black triangles represent participants that met the primary endpoint for the HALT-MS study before month 60 after transplant, and white circles represent participants that stayed in remission from active MS until the last follow-up. The line represents the mean of participants evaluated. ${ }^{*} P<0.05$, between month 0 before therapy versus months indicated after transplant using mixed model for repeated measures.

therapy, were evaluated as the cumulative proportion within $\mathrm{CD}^{+}$or $\mathrm{CD} 8^{+} \mathrm{T}$ cells in blood over time. Clones that were identified as removed from CSF (i.e., preexisting in blood and CSF that were undetectable in CSF at month 24 after transplant) formed a significantly reduced proportion of $\mathrm{CD} 4^{+}$and $\mathrm{CD} 8^{+} \mathrm{T}$ cell repertoires circulating in blood at months 12, 24, and 48 after transplant as compared with month 0 before therapy (Figure 4A and Supplemental Figure 3A). This contrasted sharply with clones that were identified as new in CSF at month 24 after transplant (i.e., preexisting in blood before therapy and undetectable in CSF before therapy), which formed a significantly greater proportion of $\mathrm{CD} 4^{+}$and $\mathrm{CD} 8^{+} \mathrm{T}$ cell repertoires circulating in blood at months 12, 24, and 48 after transplant as compared with month 0 before therapy (Figure 4B and Supplemental Figure 3B). Most of the clones that persisted in CSF at month 24 after transplant were detected in blood before therapy (Figure 2D, gray vs. blue bars); these clones transiently increased and then persisted within $\mathrm{CD}^{+}$and $\mathrm{CD}^{+} \mathrm{T}$ cell repertoires in blood after transplant (Figure $4 \mathrm{C}$ and Supplemental Figure 3C). These results show concordant effects of AHSCT therapy on clones present in both intrathecal and peripheral blood compartments.

Diversification of $T$ cell repertoires in peripheral blood and CSF following AHSCT. We next set out to determine the effects of AHSCT therapy on the degree of TCR complexity, termed TCR entropy, within and between compartments using the Shannon entropy index (8). In accordance with previous studies of hematopoietic transplantation (9), we observed earlier recovery of TCR entropy of CD4 ${ }^{+} \mathrm{T}$ cells in blood compared with their CD8 counterparts, with return to baseline entropy levels at month 24 after transplant in $\mathrm{CD}^{+}$but not $\mathrm{CD}^{+} \mathrm{T}$ cells (Figure $5 \mathrm{~A}$ ). Consistent with the reduced TCR entropy of $\mathrm{CD}^{+} \mathrm{T}$ cells compared with $\mathrm{CD}^{+} \mathrm{T}$ cells in blood at month 24 after transplant (Figure 5A and Supplemental Figure $4 \mathrm{~A}$ ), the ratio of effector memory to naive (EM/naive) $\mathrm{T}$ cells was significantly increased from month 0 before therapy at month 24 after transplant in the $\mathrm{CD}^{+}$compartment and not in the $\mathrm{CD} 4^{+}$compartment (Figure 5B and Supplemental Figure 4B).

Further participant stratification revealed an association between CMV infection after transplant and the reduced recovery of TCR entropy in circulating $\mathrm{CD}^{+} \mathrm{T}$ cells at month 24 after transplant $(P=0.003)$ (Figure 5A and Supplemental Figure 4A). This could be explained by oligoclonal expansion of CMV-reactive $\mathrm{CD}^{+} \mathrm{T}$ cells $(10)$ and potentially cytokine-mediated bystander activation of memory $\mathrm{T}$ cells (11). In support of these possibilities, CMV positivity after transplant was associated with higher ratios of central memory/naive $\mathrm{T}$ cells for $\mathrm{CD} 8^{+} \mathrm{T}$ cells at months 12 and 24 and $\mathrm{EM} /$ naive $\mathrm{T}$ cells for both $\mathrm{CD}^{+}$and $\mathrm{CD}^{+} \mathrm{T}$ cells at months 12, 24, and 36 after transplant (Figure 5B and Supplemental Figure 4B). 

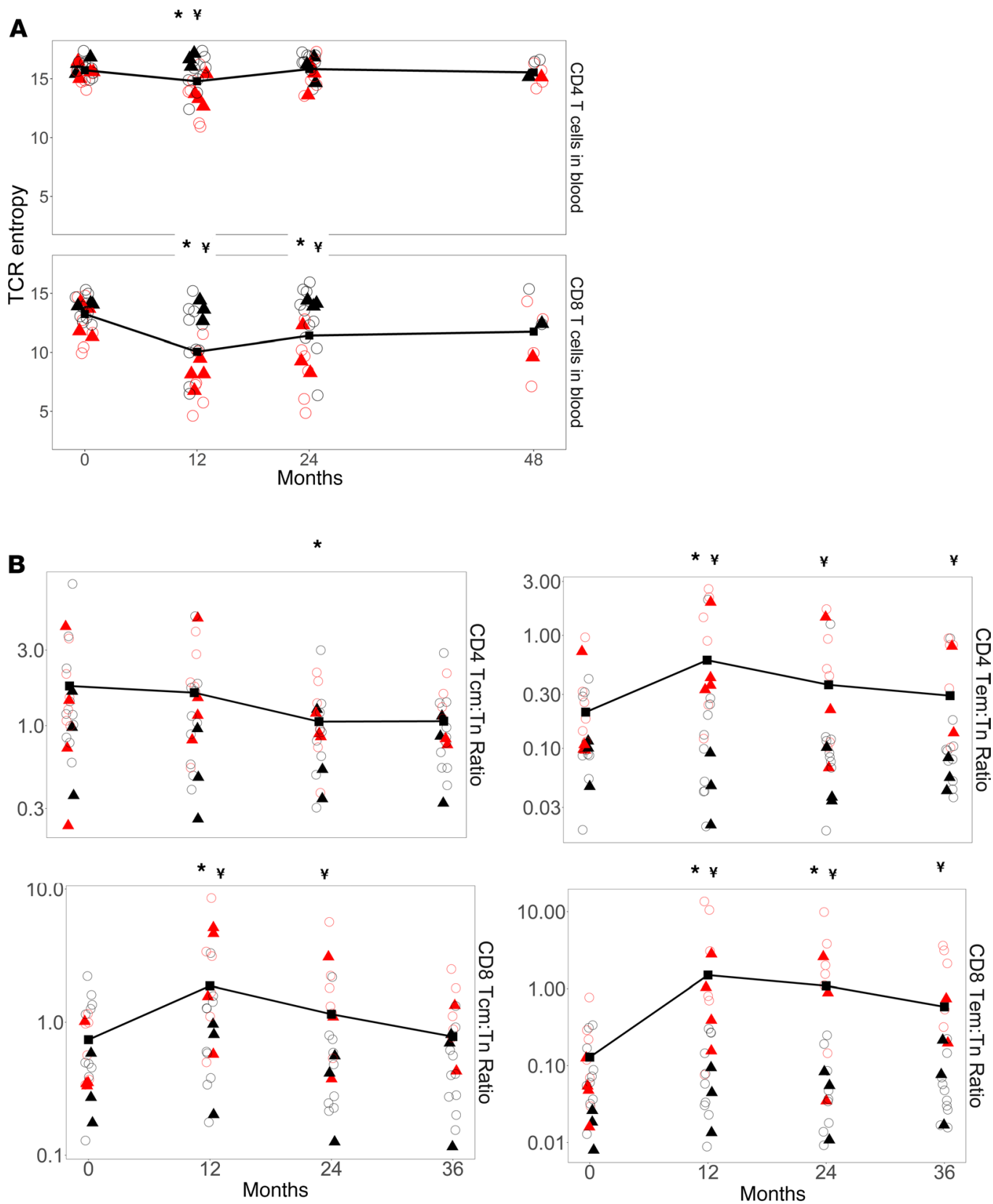

$\Delta$ Disease reactivation CMV- post-Tx Remission CMV- post-TX

$\Delta$ Disease reactivation $\mathrm{CMV}+$ post-Tx Remission CMV+ post-Tx

Figure 5. CMV infection after AHSCT therapy is associated with impaired TCR diversification and an increased ratio of memory-to-naive cell subsets in circulating T cells. (A) TCR repertoire diversity was analyzed using the Shannon entropy index in blood $\mathrm{CD}^{+}$and $\mathrm{CD}^{+} \mathrm{T}$ cells before therapy and at months 12,24 , and 48 after transplant. (B) Ratios of circulating $\mathrm{CD}^{+}$(top) and $\mathrm{CD}^{+}$(bottom) central memory (Tcm) and effector memory (Tem) to naive $(\mathrm{Tn})$ cells were analyzed by flow cytometry, as previously reported (13). Filled triangles represent participants that met the primary endpoint for the HALT-MS study before month 60 after transplant, and open circles represent participants that stayed in remission from active MS until the last follow-up. Participants were further stratified based on CMV infection status after transplant. Black shapes represent participants that were CMVand red shapes represent participants that were $\mathrm{CMV}^{+}$by PCR after transplant. The lines represent the mean of all participants evaluated at each time point. ${ }^{*} P<0.05$, between month 0 before therapy versus months indicated after transplant using mixed model for repeated measures. ${ }^{*} P<0.05$, between $C M V+$ versus $C M V$ - groups after transplant at each visit using mixed model for repeated measures. 

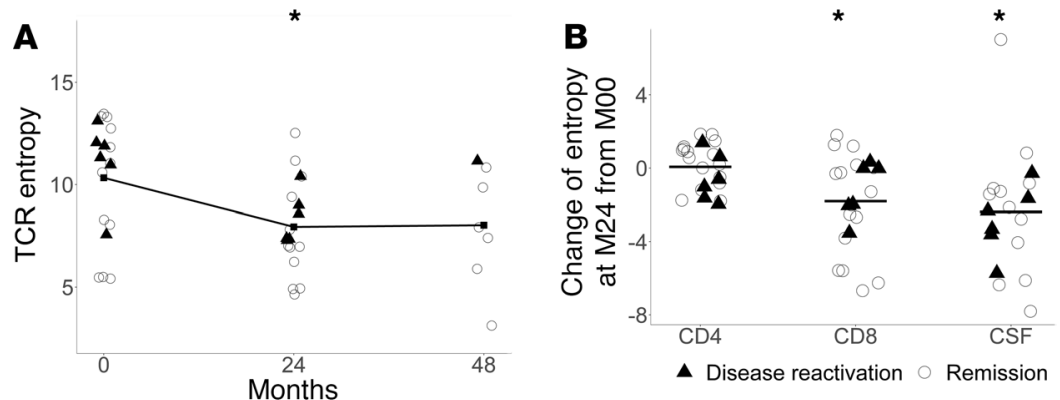

Figure 6. TCR diversification in CSF is reduced at month 24 after AHSCT therapy. (A) TCR repertoire diversity was analyzed using the Shannon entropy index in CSF before therapy and at months 24 and 48 after transplant. (B) The absolute change in TCR entropy at month 24 after transplant from baseline was calculated for CD4 ${ }^{+}$and CD8 ${ }^{+}$ T cells in blood (Figure 5A) and CSF. The lines represent the mean change from baseline at month 24 for all participants evaluated. Black triangles represent participants that met the primary endpoint for the HALT-MS study before month 60 after transplant, and white circles represent participants that stayed in remission from active MS until the last follow-up. ${ }^{*} P<0.05$, between month 0 before therapy versus at month 24 after transplant in $\mathbf{A}$ and between the absolute change in TCR entropy at month 24 (M24) from month 0 (MO0) for CD4+ $4^{+}$cells in blood compared with $\mathrm{CD}^{+} \mathrm{T}$ cells in blood or CSF cells in B.

TCR entropy was lower in the CSF repertoire (mean $10.3 \pm 2.9 \mathrm{SD}$ ) before therapy (Figure 6A and Supplemental Figure 5) as compared with $\mathrm{CD}^{+}$and $\mathrm{CD}^{+}$repertoires in blood before therapy (mean 15.7 $\pm 0.8 \mathrm{SD}$ for $\mathrm{CD}^{+}$and mean $13.2 \pm 1.5 \mathrm{SD}$ for $\mathrm{CD}^{+}$). Similar to what we observed for TCR entropy of $\mathrm{CD}^{+} \mathrm{T}$ cells at month 24 after transplant, TCR entropy of CSF was not fully reconstituted at month 24 after transplant as compared with that before therapy (Figure 6B). However, the reduced diversity of the reconstituted CSF repertoire at month 24 was not associated with CMV infection, EBV infection, age, or clinical events related to MS worsening (data not shown).

\section{Discussion}

AHSCT therapy has been shown to be effective in patients with treatment-resistant RRMS (2), yet the neuroimmunologic therapeutic mechanisms to potentially explain the strong mitigating effect on CNS inflammation seen in HALT-MS remain poorly defined. To investigate one possibility, we tested the hypothesis that the preexisting $\mathrm{T}$ cell repertoire in the intrathecal compartment before therapy was ablated and replaced with new clones after transplant. Using high-throughput TCR $\beta$ chain sequencing, we have compared the reconstitution of $\mathrm{T}$ cell repertoires in the target disease organ using CSF to paired reconstitution in peripheral blood from HALT-MS participants with active RRMS that underwent AHSCT therapy. In support of our hypothesis, we show that AHSCT therapy leads to the generation of a new $\mathrm{T}$ cell repertoire within the intrathecal compartment, providing primary evidence that immunoablation followed by AHSCT facilitates immune resetting in the CNS.

We found a greater than $90 \%$ degree of clonal ablation in the T cell repertoire in CSF that persisted out to 4 years after transplantation (Figure 2A). The reconstituted repertoires in CSF comprised predominantly new $\mathrm{T}$ cell clonotypes differentiated from engrafted aHSCs and a smaller population of mature $\mathrm{T}$ cells from the preexisting repertoire in blood that survived immunoablation (Figure 3). The robust changes we observed in relation to the CSF before therapy sharply contrast with the stability of the T cell repertoire in CSF previously reported for MS patients that underwent 2 lumbar punctures 14 months apart, where $91 \%-100 \%$ of sequences found in the first CSF sample were also found in the second sample (12). Our findings suggest that the extensive replacement was a consequence of AHSCT therapy and not simply due to physiological $\mathrm{T}$ cell turnover in CSF over time.

Interestingly, the extent of repertoire replacement was significantly greater in CSF compared with $\mathrm{CD}^{+} \mathrm{T}$ cells in blood at month $24(P<0.001)$ and in CSF compared with $\mathrm{CD}^{+} \mathrm{T}$ cells in blood at both month $24(P<0.001)$ and month $48(P<0.01)$. We speculate that the increased CD4 ${ }^{+} \mathrm{T}$ cell replacement seen in peripheral blood at 48 months (no longer significantly different from $\mathrm{T}$ cell renewal in the CSF) may reflect enlargement of the pool of new clones continuing to differentiate after year 2 . This notion is consistent with the significant increase of recent thymic emigrants we observed in the pool of $\mathrm{CD} 4^{+} \mathrm{T}$ cells in blood at month 36 after transplant compared with that before therapy (13). 
We found that $>40 \%$ of the removed CSF repertoire consisted of clonotypes that were exclusively detected in CSF (i.e., which were undetectable in peripheral blood T cells) of active RRMS patients before therapy. It is intriguing to postulate that preexisting clones enriched in CSF may be relevant to disease. Elegant studies in mice indicate that, under physiologic conditions, the choroid plexus (CP), a unique neuroimmunological interface that produces CSF and regulates immune surveillance in the CNS (14), maintains a broad repertoire of CNS-specific memory $\mathrm{CD}^{+} \mathrm{T}$ cell clones that can remain in the $\mathrm{CP}$ or enter the CSF and change effector function depending on the context of antigen presentation in the microenvironment (15). Unfortunately, the autoantigens in MS remain poorly defined, and the autoreactive repertoire may be unique among individuals with MS. Therefore, evaluation of CNS-specific T cell clones in CSF and peripheral blood specimens was beyond the scope of our current study. Our hope is that in time the autoantigens and/or TCR specificity of disease-causing T cells in MS will be revealed, so that the database of TCR $\beta$ sequencing from this study (available at https://www.itntrialshare.org/HALTMS_JCIinsight.url), and archived PBMC specimens and remaining archived PBMC specimens, will enable sufficient investigation of this possible mechanism underlying the efficacy of AHSCT.

In contrast to what was previously reported (12), our analysis demonstrated that approximately $60 \%$ of preexisting clones in CSF were detected in blood before therapy (Figure 2C). This discrepancy (mean $62 \%$ vs. 29\%) may be explained by the differences in sampling depth (ultra-deep versus survey level) and methods (sorted $\mathrm{CD}^{+}$and $\mathrm{CD}^{+} \mathrm{T}$ cells versus whole blood) used for evaluating $\mathrm{T}$ cells in blood and/or differences in the study cohorts (12). The notable percentage of overlap of CSF clones detected in blood before therapy, together with the concordant effects of AHSCT observed on clonotypes shared between compartments before therapy (Figure 4), suggests that peripheral blood could serve as a surrogate for CSF to interrogate TCR repertoire as a biomarker associated with efficacy in future studies of AHSCT therapy in active RRMS.

During the steady state, circulating leukocytes can readily enter the $\mathrm{CP}$, but few $\mathrm{T}$ cells other than CD4 T cells with a central memory phenotype enter the CSF (16). In contrast, leukocyte migration into the CSF increases as a result of decreased barrier function and augmented chemokine production during neuroinflammation (14). Therefore, we anticipated enhanced sharing of preexisting clones between immune compartments before therapy in active RRMS and postulated that the degree of sharing and repertoire complexity would be reduced following the attenuation of inflammation and normalization of leukocyte trafficking into the CNS through the CSF after transplant. Several observations support this possibility. First, the percentage of CSF clones detected in blood was significantly decreased at month 24 after transplant compared with that before therapy (Figure 2C). Second, clones removed from CSF were present in blood but at reduced frequencies at months 12, 24, and 48 after transplant compared with that before therapy (Figure 4A). Third, diversification of the TCR repertoire in CSF was not fully reconstituted to pretherapy levels at month 24 after transplant (Figure 6). This could be explained by reduced trafficking of $\mathrm{T}$ cells into the $\mathrm{CNS}$ as a result of restored blood-brain barrier function and/ or restoration of $\mathrm{T}$ cell homing programs after immunoablation and reconstitution. Alternatively, the reduced complexity of the CSF repertoire could result from increased clonal dominance in association with recurrent autoimmunity; however, we believe that this is less likely given the positive long-term outcome of most participants in the HALT-MS trial (2).

Our interim analysis suggested that improved clinical outcomes were associated with higher TCR diversity of T cells in peripheral blood at month 2 after transplant (3). Given that this association was not observed at month 12 after transplant, we did not expect to find an association between clinical outcome and diversification of the TCR repertoire in blood T cells at months 24 and 48 analyzed here. While none of our TCR repertoire analyses revealed associations with disparate long-term clinical outcomes, we accept the limitations of the study for identifying biomarkers of response as reported previously (13).

Taken together, our findings support the hypothesis that AHSCT therapy removes the majority of $\mathrm{T}$ cells existing in the intrathecal compartment of patients with active RRMS, likely, including pathogenic $\mathrm{T}$ cells, and induces the generation of a newly differentiated immune repertoire that persists in both intrathecal and peripheral blood compartments. Of the preexisting clones in CSF, approximately $60 \%$ were also detected in before therapy blood, and concordant treatment effects were observed for clonotypes in both compartments following AHSCT. These results indicate that replacement of the preexisting TCR repertoire in active RRMS is a mechanism for AHSCT efficacy and suggest that peripheral blood could serve as a surrogate for CSF to define mechanisms associated with efficacy in future studies of AHSCT. 
Our potentially novel findings identify avenues of investigation for future studies of AHSCT therapy and other immunomodulatory intervention strategies for MS.

\section{Methods}

Further information can be found in the Supplemental Methods.

Research specimens. Of the 24 transplanted HALT-MS participants that had paired CSF and peripheral blood specimens for evaluation before therapy, 18 had paired CSF and peripheral blood specimens for evaluation at month 24 after transplant, and 7 of these participants had specimens for evaluation at month 48 after transplant. Our analyses included specimens from a subgroup of 7 participants who did not meet the trial definition of success of therapy. However, no blood or CSF samples were obtained at the time of MS reactivation (i.e., at the time of relapse, new MRI activity, or worsening of disability after transplantation).

Cell preparation and DNA isolation. PBMCs frozen in 20\% DMSO/human AB serum were thawed and then separated by sequential positive selections for $\mathrm{CD}^{+}$cells (Miltenyi Biotec; 130-045-101) followed by $\mathrm{CD}^{+}$cells (Miltenyi Biotec; 130-045-201) per the manufacturer's instructions. Frozen cellular pellets from 20-30 mL CSF were thawed for genomic DNA isolation. DNA was isolated using the Qiagen QIAmp DNA mini kit (Qiagen; 51306) per the manufacturer's instructions, except the elution volume was increased to $100 \mu \mathrm{L}$ for use in TCR sequencing.

TCR sequencing. Due to the multicenter logistics of the HALT-MS trial, together with the limited number and stability of cells in CSF ex vivo, the decision was made to use the entire cell fraction for molecular studies. Cell counts were obtained by the clinical labs, but $\mathrm{T}$ cell counts were not and could not be estimated from the version of the Adaptive TCR $\beta$ sequencing platform (Adaptive Biotechnologies) that was used for this project. Input DNA from the cellular fraction (mean 154,000 total leukocytes) from 20-30 mL CSF collected before therapy and at months 24 and 48 after transplant was approximately $1 \mu \mathrm{g}$ per sample, and the input DNA for 1 million sorted $\mathrm{CD}^{+}$and $\mathrm{CD} 8^{+} \mathrm{T}$ cells from blood before therapy and at months 12,24 , and 48 after transplant was $5 \mu \mathrm{g}$. Rearranged TCR $\beta$ chains from all specimens were amplified, sequenced, and processed using the immunoSEQ Assay (Adaptive Biotechnologies) (17). This sequencing strategy is sufficient to achieve at least 4-fold coverage of every original template, which is sufficient to prevent a sampling effect (18). Survey-level TCR $\beta$ sequencing was used to evaluate the full complexity of the CSF repertoire (12), which is limited in T cell numbers and richness compared with blood. This produced an average of 447,387 sequence reads distributed among an average of 4,275 unique TCRB CDR3 rearrangements for each CSF cellular pellet sample (Supplemental Table 1). Ultradeep level TCR $\beta$ sequencing was used to enable accurate description of richer repertoires and detection of low-frequency clones in the millions of $\mathrm{CD}^{+}$and $\mathrm{CD} 8^{+} \mathrm{T}$ cells sorted from blood of individual participants for longitudinal evaluation (19). This produced an average of 6,216,316 and 5,911,245 sequence reads, distributed among an average of 210,455 and 112,709 unique TCRB CDR3 rearrangements, for each sample of 1 million $\mathrm{CD}^{+}$or $\mathrm{CD}^{+} \mathrm{T}$ cells (Supplemental Table 2). For known autoreactive clones in psoriasis, their abundance in the peripheral blood is near or above $1 /$ million cells (19). We sequenced 1 million $\mathrm{T}$ cells from each of the $\mathrm{CD}^{+}$and $\mathrm{CD}^{+}$compartments, reaching levels far below $1 /$ million PBMCs (19). Sequence comparisons were based on annotated TCR $\beta$ variable (TCRBV) and TCR $\beta$ joining (TCRBJ) gene segments and CDR3 aa sequence. For each repertoire, the frequencies of clones with identical TCRBV, TCRBJ, and CDR3 aa sequences but different DNA sequences were merged. The assumption is that $\mathrm{T}$ cell clones with identical TCRBV, TCRBJ, and CDR3 aa sequences recognize the same antigen. CDR3 aa sequences were favored over DNA sequences to better reflect changes in antigen recognition of the reconstituted $\mathrm{T}$ cell repertoire between time points. Shared clones between 2 repertoires are defined as having identical TCRBV and TCRBJ segments and CDR3 aa sequence.

To determine the overlap between $\mathrm{T}$ cell repertoires from identical tissues at different time points, we used the TCR overlap percentage (8). The percentage of overlap is weighted by the relative frequencies of shared clones between time points, ensuring that this method is robust to differences in sampling depth. The massive difference in numbers of $\mathrm{T}$ cell clones sampled from peripheral blood compared with the CSF precluded the use of this method to compare the overlap of these tissues. Instead, the proportion of specific clonotypes was tracked from 1 compartment to the other before therapy or over time. The Shannon entropy index was used as an index of TCR repertoire diversity. This method weights clones by relative frequency, favoring neither dominant or rare clones disproportionately. 
Data and material availability. Data sets for these analyses are accessible through TrialShare, a public website managed by the Immune Tolerance Network (https://www.itntrialshare.org/HALTMS_JCIinsight.url), which allows the user to filter the underlying data and generate figures and results of analysis in addition to those submitted as part of the manuscript.

Statistics. For longitudinal assessment between time points, data were analyzed using the mixed model for repeated measures. Pairwise comparisons were made between time points, and $P$ values were adjusted using Tukey's multiple testing corrections. The percentage of CSF clones detected in blood at month 0 before therapy was compared to the percentage of CSF clones detected in blood at month 24 after transplant using a paired 2-tailed $t$ test. R 3.5.1 was used for all analyses. Statistical significance was set at $5 \%(P<0.05)$.

Study approval. The HALT-MS study (a phase II study of high-dose immunosuppressive therapy and autologous $\mathrm{CD}_{3} 4^{+}$hematopoietic stem cell transplant for the treatment of poor prognosis MS) was sponsored by National Institute of Allergy and Infectious Diseases and conducted by the Immune Tolerance Network (ITN033AI) (ClinicalTrials.gov NCT00288626). The protocol was reviewed and approved by the IRB at each of the clinical sites (University of Washington, Seattle, Washington, USA; Baylor College of Medicine, Houston, Texas, USA; MD Anderson Cancer Center, Houston, Texas, USA; The Ohio State University Medical Center, Columbus, Ohio, USA), and all subjects provided informed consent prior to their participation. This prospective, open-label, single-arm, multicenter clinical trial was designed to determine the 5-year durability of disease stabilization in MS subjects after high-dose immunosuppressive therapy and AHSCT (2).

\section{Author contributions}

RAN, LMG, and PAM were responsible for clinical study design, conduct, supervision, and coordination. $\mathrm{KMH}, \mathrm{NL}, \mathrm{PL}, \mathrm{HR}, \mathrm{LAT}$, and PAM designed the experiment, presented and analyzed the data, and interpreted the data. $\mathrm{KMH}$ and PAM drafted the manuscript. All authors contributed to the critical revision of the manuscript for important intellectual content.

\section{Acknowledgments}

The authors thank their colleagues at the Immune Tolerance Network and their collaborators who contribute in many capacities to Immune Tolerance Network projects and perspectives. The authors are grateful to the ITN033AI HALT-MS study participants, and thank the clinical site investigators and study coordinators. Research reported in this publication was conducted by the Immune Tolerance Network and sponsored by the National Institute of Allergy and Infectious Diseases of the NIH under award UM1AI109565. We are grateful for support from the UK National Institute of Health Research (NIHR) Biomedical Research Centre funding scheme (to PAM). The content is solely the responsibility of the authors and does not necessarily represent the official views of the NIH, NIHR, or the United Kingdom Department of Health.

Address correspondence to: Paolo A. Muraro, Department of Brain Sciences, Imperial College London, Burlington Danes Building, Du Cane Road, Hammersmith Hospital Campus, London W12 0NN, United Kingdom. Phone: 44.207.594.6670; Email: p.muraro@imperial.ac.uk.

1. Alexander T, Arnold R, Hiepe F, Radbruch A. Resetting the immune system with immunoablation and autologous haematopoietic stem cell transplantation in autoimmune diseases. Clin Exp Rheumatol. 2016;34(4 Suppl 98):53-57.

2. Nash RA, et al. High-dose immunosuppressive therapy and autologous HCT for relapsing-remitting MS. Neurology. 2017;88(9):842-852

3. Muraro PA, et al. T cell repertoire following autologous stem cell transplantation for multiple sclerosis. J Clin Invest. 2014;124(3):1168-1172.

4. Babbe $\mathrm{H}$, et al. Clonal expansions of CD8(+) T cells dominate the $\mathrm{T}$ cell infiltrate in active multiple sclerosis lesions as shown by micromanipulation and single cell polymerase chain reaction. J Exp Med. 2000;192(3):393-404.

5. Wilson EH, Weninger W, Hunter CA. Trafficking of immune cells in the central nervous system. J Clin Invest. 2010;120(5):1368-1379.

6. Stangel M, Fredrikson S, Meinl E, Petzold A, Stüve O, Tumani H. The utility of cerebrospinal fluid analysis in patients with multiple sclerosis. Nat Rev Neurol. 2013;9(5):267-276.

7. Cepok S, et al. Patterns of cerebrospinal fluid pathology correlate with disease progression in multiple sclerosis. Brain. 2001;124(Pt 11):2169-2176.

8. Emerson RO, et al. High-throughput sequencing of T-cell receptors reveals a homogeneous repertoire of tumour-infiltrating lymphocytes in ovarian cancer. J Pathol. 2013;231(4):433-440.

9. van Heijst JW, et al. Quantitative assessment of T cell repertoire recovery after hematopoietic stem cell transplantation. Nat Med. 2013;19(3):372-377. 
10. Suessmuth Y, et al. CMV reactivation drives posttransplant T-cell reconstitution and results in defects in the underlying TCR $\beta$ repertoire. Blood. 2015;125(25):3835-3850.

11. Doisne JM, et al. CD8+ T cells specific for EBV, cytomegalovirus, and influenza virus are activated during primary HIV infection. J Immunol. 2004;173(4):2410-2418.

12. Lossius A, et al. High-throughput sequencing of TCR repertoires in multiple sclerosis reveals intrathecal enrichment of EBV-reactive CD8+ T cells. Eur J Immunol. 2014;44(11):3439-3452.

13. Harris KM, Lu T, Lim N, Turka LA. Challenges and opportunities for biomarkers of clinical response to AHSCT in autoimmunity. Front Immunol. 2018;9:100.

14. Lopes Pinheiro MA, et al. Immune cell trafficking across the barriers of the central nervous system in multiple sclerosis and stroke. Biochim Biophys Acta. 2016;1862(3):461-471.

15. Baruch K, et al. CNS-specific immunity at the choroid plexus shifts toward destructive Th2 inflammation in brain aging. Proc Natl Acad Sci USA. 2013;110(6):2264-2269.

16. Kivisäkk P, et al. Human cerebrospinal fluid central memory CD4+ T cells: evidence for trafficking through choroid plexus and meninges via P-selectin. Proc Natl Acad Sci USA. 2003;100(14):8389-8394.

17. Robins H, et al. Ultra-sensitive detection of rare T cell clones. J Immunol Methods. 2012;375(1-2):14-19.

18. Zhang L, Bertucci AM, Ramsey-Goldman R, Burt RK, Datta SK. Regulatory T cell (Treg) subsets return in patients with refractory lupus following stem cell transplantation, and TGF-beta-producing CD8+ Treg cells are associated with immunologica remission of lupus. J Immunol. 2009;183(10):6346-6358.

19. Matos TR, et al. Clinically resolved psoriatic lesions contain psoriasis-specific IL-17-producing $\alpha \beta$ T cell clones. J Clin Invest 2017;127(11):4031-4041. 